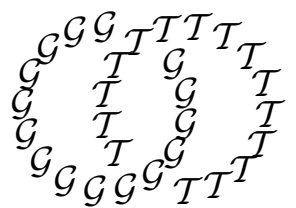

\title{
A proof of Atiyah's conjecture on configurations of four points in Euclidean three-space
}

\author{
Michael Eastwood \\ PAUl Norbury \\ Pure Mathematics Department \\ Adelaide University \\ South Australia 5005
}

Email: meastwoo@maths.adelaide.edu.au, pnorbury@maths.adelaide.edu.au

\begin{abstract}
From any configuration of finitely many points in Euclidean three-space, Atiyah constructed a determinant and conjectured that it was always non-zero. In this article we prove the conjecture for the case of four points.
\end{abstract}

\section{AMS Classification numbers Primary: 51M04}

Secondary: $70 \mathrm{G} 25$

Keywords: Atiyah's conjecture, configuration space

Proposed: Walter Neumann

Seconded: Ralph Cohen, Steven Ferry

(C) Geometry $\& \mathcal{G}$ Topology $\mathcal{P}$ ublications
Received: 26 October 2001

Revised: 10 November 2001 
Consider $n$ distinct points in Euclidean three-space. Fixing attention on one of these points, the others give rise to $n-1$ points on its sphere of vision. Thinking of this as the Riemann sphere gives a monic polynomial of degree $\leq n-1$, having as its zeroes the points not equal to the point chosen to be $\infty$. We may regard its coefficients as a complex $n$-vector (for degree $d<n-1$, its first $n-1-d$ coefficients are deemed to be zero). Repeating this exercise for each of the $n$ points gives $n$ such vectors and hence an $n \times n$ matrix. In [1, 2], Atiyah conjectured that a matrix constructed in this way cannot be singular. In [3], Atiyah and Sutcliffe amass a great deal of numerical evidence for this conjecture and formulate a series of further conjectures based on the geometry that their numerical studies apparently reveal.

In spite of overwhelming evidence in its favour, the basic conjecture, as stated above, remains surprisingly resistant. The case $n=3$ is not too hard: a geometric argument is given in [1] and an algebraic one in [2]. In this article we establish the case $n=4$.

\section{Normalisation}

In describing Atiyah's conjecture above we used only the directions defined by pairs of points amongst the $n$ points. It turns out to be far more natural to keep track of scale as well as direction, in particular in order to see what happens if we rotate the sphere of directions in $\mathbb{R}^{3}$, obtaining a different identification with the Riemann sphere. We will use the Hopf mapping

$$
\mathbb{C}^{2} \ni\left(\begin{array}{c}
w_{1} \\
w_{2}
\end{array}\right) \stackrel{h}{\longmapsto}\left(\begin{array}{c}
\left(\left|w_{1}\right|^{2}-\left|w_{2}\right|^{2}\right) / 2 \\
w_{1} \bar{w}_{2}
\end{array}\right) \in \mathbb{R} \times \mathbb{C} \cong \mathbb{R}^{3}
$$

as follows. This mapping intertwines the action of $\mathrm{SU}(2)$ on $\mathbb{C}^{2}$ with the action of $\mathrm{SO}(3)$ on $\mathbb{R}^{3}$ and descends to an isomorphism from $\mathbb{C P}_{1}$ to the sphere of rays through the origin in $\mathbb{R}^{3}$. Therefore, for each point in $\mathbb{R}^{3} \backslash\{0\}$, we may choose a corresponding point in $\mathbb{C}^{2} \backslash\{0\}$ defined up to phase. Their symmetric tensor product lies in

$$
\bigodot^{n-1} \mathbb{C}^{2} \cong \mathbb{C}^{n}
$$

and is also well-defined up to phase. We may regard this as normalising, up to phase, the complex $n$-vectors appearing in our initial formulation of Atiyah's conjecture. If we now construct the columns of an $n \times n$ matrix $M$ in this way, then $\operatorname{det} M$ is well-defined up to phase and $|\operatorname{det} M|^{2}$ is invariant under Euclidean motions. 
Following Atiyah and Sutcliffe [3], we may normalize $\operatorname{det} M$ further. Consider the mapping

$$
\mathbb{C}^{2} \ni\left(\begin{array}{c}
w_{1} \\
w_{2}
\end{array}\right) \stackrel{\sigma}{\longmapsto}\left(\begin{array}{c}
-\bar{w}_{2} \\
\bar{w}_{1}
\end{array}\right) \in \mathbb{C}^{2}
$$

observing that $h(\sigma(w))=-h(w)$ for all $w \in \mathbb{C}^{2}$. Also note that

$$
\sigma\left(e^{i \theta} w\right)=e^{-i \theta} \sigma(w) \quad \text { and } \quad \sigma(\sigma(w))=-w .
$$

Fix an ordering for our original $n$ points in $\mathbb{R}^{3}$. Each pair of these points contributes twice to $\operatorname{det} M$, once when the later point is viewed from the earlier and once when this view is reversed. We mandate using $w$ and $\sigma(w)$, respectively, in lifting to $\mathbb{C}^{2}$. By virtue of (1), both the phase ambiguity $w \mapsto e^{i \theta} w$ and the ordering ambiguity cancel from $\operatorname{det} M$. In conclusion, $\operatorname{det} M$ is invariant under Euclidean motions. It is easy to check that $\operatorname{det} M$ is replaced by its complex conjugate under reflection. In particular, if all points lie in a plane then the determinant is real. For further details see $[2,3]$. We shall call det $M$, normalised in this way, the Atiyah determinant. In [3] a scale invariant normalisation $D$ is used. The two normalisations are related by

$$
\operatorname{det} M=D \cdot \prod_{i>j}\left(2 r_{i j}\right)
$$

where $r_{i j}$ is the distance between the $i^{\text {th }}$ and $j^{\text {th }}$ points.

We are free to use Euclidean motions to place points in convenient locations. Let us do this to verify the conjecture when $n=3$, choosing the three points in $\mathbb{R} \times \mathbb{C}$ to be

$$
\left(\begin{array}{l}
0 \\
0
\end{array}\right) \quad\left(\begin{array}{l}
0 \\
a
\end{array}\right) \quad\left(\begin{array}{l}
0 \\
z
\end{array}\right)
$$

with $a$ real. They form a triangle with side lengths $a, b=|z|$, and $c=|a-z|$. We may use the following

$$
\begin{aligned}
& \frac{1}{\sqrt{a}}\left(\begin{array}{l}
a \\
a
\end{array}\right) \stackrel{h}{\mapsto}\left(\begin{array}{c}
0 \\
a
\end{array}\right) \quad \frac{1}{\sqrt{b}}\left(\begin{array}{l}
b \\
\bar{z}
\end{array}\right) \stackrel{h}{\mapsto}\left(\begin{array}{l}
0 \\
z
\end{array}\right) \\
& \frac{1}{\sqrt{a}}\left(\begin{array}{c}
-a \\
a
\end{array}\right) \stackrel{h}{\mapsto}\left(\begin{array}{c}
0 \\
-a
\end{array}\right) \quad \frac{1}{\sqrt{c}}\left(\begin{array}{c}
c \\
\bar{z}-a
\end{array}\right) \stackrel{h}{\mapsto}\left(\begin{array}{c}
0 \\
z-a
\end{array}\right) \\
& \frac{1}{\sqrt{b}}\left(\begin{array}{c}
-z \\
b
\end{array}\right) \stackrel{h}{\mapsto}\left(\begin{array}{c}
0 \\
-z
\end{array}\right) \quad \frac{1}{\sqrt{c}}\left(\begin{array}{c}
a-z \\
c
\end{array}\right) \stackrel{h}{\mapsto}\left(\begin{array}{c}
0 \\
a-z
\end{array}\right)
\end{aligned}
$$

Geometry ${ }^{6}$ Topology, Volume 5 (2001) 
in computing $\operatorname{det} M$. We obtain

$$
\begin{gathered}
\frac{1}{a b c}\left|\begin{array}{ccc}
a b & -a c & -z(a-z) \\
a \bar{z}+a b & -a(\bar{z}-a)+a c & -z c+b(a-z) \\
a \bar{z} & a(\bar{z}-a) & b c
\end{array}\right| \\
=a((z+\bar{z})(c-a-b)+2 b(a+b+3 c)) \\
=\left(a^{2}+b^{2}-c^{2}\right)(c-a-b)+2 a b(a+b+3 c) \\
=d_{3}(a, b, c)+8 a b c,
\end{gathered}
$$

where

$$
d_{3}(a, b, c)=(a+b-c)(b+c-a)(c+a-b) .
$$

The triangle inequalities imply that $d_{3}(a, b, c) \geq 0$ with equality if and only if the points lie on a line. Therefore $\operatorname{det} M \geq 8 a b c>0$ and, in particular, is non-zero, as required.

\section{The case $n=4$}

Theorem 1 For any four points in $\mathbb{R}^{3}$, the Atiyah determinant is non-zero.

Proof Choose the four points in $\mathbb{R}^{3}=\mathbb{R} \times \mathbb{C}$ to be

$$
\left(\begin{array}{c}
0 \\
z_{1}
\end{array}\right) \quad\left(\begin{array}{c}
0 \\
z_{2}
\end{array}\right) \quad\left(\begin{array}{c}
0 \\
z_{3}
\end{array}\right) \quad\left(\begin{array}{c}
r \\
0
\end{array}\right)
$$

Put $z_{i j}=z_{i}-z_{j}$ for $i>j$ and label the distances between points by $r_{i j}$. We define $z_{4}=0$ so that $z_{4 j}=-z_{j}$. Thus, $r_{i j}^{2}=\left|z_{i j}\right|^{2}$ for $i<4$ and $r_{4 j}^{2}=$ $r^{2}+\left|z_{4 j}\right|^{2}$.

For $j<i<4$ the vector running from the $j^{\text {th }}$ point to the $i^{\text {th }}$ point may be lifted to

$$
w=\frac{1}{\sqrt{r_{i j}}}\left(\begin{array}{c}
r_{i j} \\
\bar{z}_{i j}
\end{array}\right), \text { with } \sigma(w)=\frac{1}{\sqrt{r_{i j}}}\left(\begin{array}{c}
-z_{i j} \\
r_{i j}
\end{array}\right) .
$$

Similarly, if we put $R_{4 j}=r_{4 j}+r$ for $j<4$, then

$$
w=\frac{1}{\sqrt{R_{4 j}}}\left(\begin{array}{c}
R_{4 j} \\
\bar{z}_{4 j}
\end{array}\right) \text { and } \sigma(w)=\frac{1}{\sqrt{R_{4 j}}}\left(\begin{array}{c}
-z_{4 j} \\
R_{4 j}
\end{array}\right)
$$

lift to $\mathbb{C}^{2}$ the vectors in $\mathbb{R}^{3}$ joining the $j^{\text {th }}$ point to the $4^{\text {th }}$ point and vice versa. For each of the four points, the coefficients of the corresponding third 
degree polynomial give the following four vectors:

$$
\begin{gathered}
v_{1}=\frac{1}{\sqrt{r_{21} r_{31} R_{41}}}\left(\begin{array}{c}
r_{21} r_{31} R_{41} \\
r_{21} r_{31} \bar{z}_{41}+r_{21} R_{41} \bar{z}_{31}+r_{31} R_{41} \bar{z}_{21} \\
r_{21} \bar{z}_{31} \bar{z}_{41}+r_{31} \bar{z}_{21} \bar{z}_{41}+R_{41} \bar{z}_{21} \bar{z}_{31} \\
\bar{z}_{21} \bar{z}_{31} \bar{z}_{41}
\end{array}\right) \\
v_{2}=\frac{1}{\sqrt{r_{32} R_{42} r_{21}}}\left(\begin{array}{c}
-r_{32} R_{42} z_{21} \\
-r_{32} z_{21} \bar{z}_{42}+r_{32} R_{42} r_{21}-z_{21} R_{42} \bar{z}_{32} \\
r_{32} r_{21} \bar{z}_{42}-z_{21} \bar{z}_{32} \bar{z}_{42}+R_{42} \bar{z}_{32} r_{21} \\
\bar{z}_{32} r_{21} \bar{z}_{42}
\end{array}\right) \\
v_{3}=\frac{1}{\sqrt{R_{43} r_{31} r_{32}}}\left(\begin{array}{c}
z_{31} z_{32} R_{43} \\
z_{31} z_{32} \bar{z}_{43}-z_{31} R_{43} r_{32}-z_{32} R_{43} r_{31} \\
-z_{31} r_{32} \bar{z}_{43}-z_{32} r_{31} \bar{z}_{43}+R_{43} r_{31} r_{32} \\
r_{31} r_{32} \bar{z}_{43} \\
-z_{41} z_{42} z_{43}
\end{array}\right) \\
v_{4}=\frac{1}{\sqrt{R_{41} R_{42} R_{43}}}\left(\begin{array}{c}
z_{41} z_{42} R_{43}+z_{41} z_{43} R_{42}+z_{42} z_{43} R_{41} \\
-z_{41} R_{42} R_{43}-z_{42} R_{41} R_{43}-z_{43} R_{41} R_{42} \\
R_{41} R_{42} R_{43}
\end{array}\right)
\end{gathered}
$$

and we may take $M$ to be the matrix with column vectors $v_{i}$. Hence,

$$
\operatorname{det} M=P /\left(r_{21} r_{31} r_{32} R_{41} R_{42} R_{43}\right)
$$

where $P$ is a polynomial consisting of monomials each of which contains one of $r_{i j}^{2}, r_{i j} z_{i j}, r_{i j} \bar{z}_{i j}$, or $z_{i j} \bar{z}_{i j}$ for each $j<i<4$, and one of $R_{4 j}^{2}, R_{4 j} z_{4 j}$, $R_{4 j} \bar{z}_{4 j}$, or $z_{4 j} \bar{z}_{4 j}$. Since $z_{i j} \bar{z}_{i j}=r_{i j}^{2}$, each monomial is divisible by $r_{i j}$ and, since $\left|z_{4 j}\right|^{2}=R_{4 j}\left(r_{4 j}-r\right)$, each monomial is divisible by $R_{4 j}$. Therefore, we can divide by the factor of $r_{21} r_{31} r_{32} R_{41} R_{42} R_{43}$ leaving monomials with one of $r_{i j}, z_{i j}$ or $\bar{z}_{i j}$ for each $j<i<4$, and one of $\left(r_{4 j}+r\right), z_{4 j}, \bar{z}_{4 j}$ or $\left(r_{4 j}-r\right)$. It follows that $\operatorname{det} M$ is now expressed as a homogeneous degree 6 polynomial in $r, r_{i j}, z_{i j}$ and $\bar{z}_{i j}$ for $j<i \leq 4$.

Recall that $\operatorname{det} M$ is invariant under Euclidean motions. Moreover, the six distances $r_{i j}$ determine our configuration of four points. Also, notice that these distances are constrained only by triangle inequalities. Hence, the Atiyah determinant det $M$ may be regarded as a function of the independent variables $r_{i j}$. We claim that

- $\Re(\operatorname{det} M)$ is a polynomial in $r_{i j}$ (homogeneous of degree 6).

- $|\operatorname{det} M|^{2}$ is a polynomial in $r_{i j}$ (homogeneous of degree 12). 
Having done this we shall use the triangle inequalities on the four faces of our configuration to show that, in fact, $\Re(\operatorname{det} M)>0$. This is more than sufficient to finish the proof.

It is convenient to set $z_{i j}=-z_{j i}$ and $r_{i j}=r_{j i}$ when $j>i$. The monomials in our expression for det $M$ contain an equal number of $z_{i j}$ and $\bar{z}_{k l}$. Consider the product $z_{i j} \bar{z}_{k l}$. There are two cases:

(i) $\{i, j\}$ and $\{k, l\}$ have an element in common (suppose $l=j$ ):

$$
z_{i j} \bar{z}_{k j}=(1 / 2)\left(r_{i j}^{2}+r_{k j}^{2}-r_{k i}^{2}\right)+2 A_{i j k} \sqrt{-1}
$$

where $A_{i j k}$, defined by (3), equals plus or minus the area of the $i j k^{\text {th }}$ triangle under the projection $\mathbb{R}^{3}=\mathbb{R} \times \mathbb{C} \rightarrow \mathbb{C}$ onto the complex plane;

(ii) $\{i, j, k, l\}=\{1,2,3,4\}$ :

$$
z_{i j} \bar{z}_{k l}=z_{i j} \bar{z}_{k j} z_{k j} \bar{z}_{k l} / r_{k j}^{2}
$$

and we can rewrite the numerator as in (i).

We claim that all quadratic expressions in the $A_{i j k}$ may be written as polynomials in the $r_{i j}$ and $r^{2}$. Specifically, when all four points lie in the complex plane, then one may verify that

$$
16 A_{i j k} A_{i j l}=2 r_{i j}^{2}\left(r_{i k}^{2}+r_{i l}^{2}-r_{k l}^{2}\right)-\left(r_{i j}^{2}+r_{i k}^{2}-r_{j k}^{2}\right)\left(r_{i j}^{2}+r_{i l}^{2}-r_{j l}^{2}\right)
$$

and when the fourth point lies off the plane $(r>0)$, we replace $r_{4 j}^{2}$ by $r_{4 j}^{2}-r^{2}$.

Now, observe that our formulae so far for $\Re(\operatorname{det} M)$ and $(\Im(\operatorname{det} M))^{2}$ involve only quadratics expressions in $A_{i j k}$. If we substitute according to (4) and its non-planar version, we obtain rational expressions for $\Re(\operatorname{det} M)$ and $(\Im(\operatorname{det} M))^{2}$ in the seven quantities in $r_{i j}$ and $r$, the denominator being a polynomial in the six variables $r_{i j}$. Recall that a reflection such as $r \mapsto-r$ conjugates $\operatorname{det} M$. Hence, we may drop all odd powers of $r$ in the numerators, to obtain polynomials in $r_{i j}$ and $r^{2}$.

Finally, we eliminate $r^{2}$ from these expressions. This is possible by writing the volume $V$ of the tetrahedron with vertices our four points in two different ways. On the one hand

$$
\begin{aligned}
144 V^{2}= & -r_{21}^{4} r_{43}^{2}-r_{21}^{2} r_{43}^{4}-r_{32}^{2} r_{41}^{4}-r_{32}^{4} r_{41}^{2}-r_{31}^{4} r_{42}^{2}-r_{31}^{2} r_{42}^{4} \\
& +r_{21}^{2} r_{43}^{2} r_{31}^{2}+r_{21}^{2} r_{43}^{2} r_{41}^{2}-r_{21}^{2} r_{42}^{2} r_{41}^{2}+r_{21}^{2} r_{42}^{2} r_{43}^{2} \\
& +r_{21}^{2} r_{42}^{2} r_{31}^{2}+r_{21}^{2} r_{32}^{2} r_{43}^{2}-r_{21}^{2} r_{32}^{2} r_{31}^{2}+r_{32}^{2} r_{42}^{2} r_{41}^{2} \\
& +r_{31}^{2} r_{42}^{2} r_{41}^{2}+r_{32}^{2} r_{43}^{2} r_{41}^{2}-r_{32}^{2} r_{42}^{2} r_{43}^{2}+r_{32}^{2} r_{42}^{2} r_{31}^{2} \\
& +r_{31}^{2} r_{42}^{2} r_{43}^{2}+r_{32}^{2} r_{31}^{2} r_{41}^{2}-r_{31}^{2} r_{43}^{2} r_{41}^{2}+r_{21}^{2} r_{32}^{2} r_{41}^{2} .
\end{aligned}
$$


On the other hand, let $A$ denote the area of the triangular base in $\mathbb{C}$. Then

$$
16 A^{2}=2 r_{21}^{2} r_{31}^{2}+2 r_{21}^{2} r_{32}^{2}+2 r_{31}^{2} r_{32}^{2}-r_{21}^{4}-r_{31}^{4}-r_{32}^{4}
$$

and $V=r A / 3$. We may therefore replace $r^{2}$ by $9 V^{2} / A^{2}$, as required.

Thus, we may conclude that $\Re(\operatorname{det} M)$ and $(\Im(\operatorname{det} M))^{2}$ are rational functions of the variables $r_{i j}$ but, if we now clear any common factors, we claim they are, in fact, polynomials. To see this, notice that in (ii) we had a choice when we introduced $\bar{z}_{k j} z_{k j} / r_{k j}^{2}$. We could have insisted that $\{k, j\} \subset\{1,2,3\}$. Then the denominators of $\Re(\operatorname{det} M)$ and $(\Im(\operatorname{det} M))^{2}$ would not involve $r_{4 j}$. However, $\operatorname{det} M$ does not see the ordering of our four points. Hence, if $r_{4 j}$ are omitted from the denominators, then so are all variables $r_{i j}$, as required.

It remains to calculate these polynomials. We did this using Maple ${ }^{\sharp}$ and found that $\Re(\operatorname{det} M)$ is a homogeneous polynomial of degree 6 with 226 terms and $|\operatorname{det} M|^{2}$ is a homogeneous degree 12 polynomial with 4500 terms. We claim that $\Re(\operatorname{det} M)>0$. To see this, we can rewrite the output of the Maple calculation as follows:

$$
\begin{aligned}
\Re(\operatorname{det} M)= & 64 r_{21} r_{31} r_{32} r_{41} r_{42} r_{43}-4 d_{3}\left(r_{21} r_{43}, r_{31} r_{42}, r_{32} r_{41}\right) \\
& +12 \text { av }\left(r_{41}\left(\left(r_{42}+r_{43}\right)^{2}-r_{32}^{2}\right) d_{3}\left(r_{21}, r_{31}, r_{32}\right)\right)+288 V^{2} .
\end{aligned}
$$

Here, $d_{3}(a, b, c)$ is the polynomial (2) and av denotes the operation of averaging a polynomial in $r_{i j}$ under the action of $\mathcal{S}_{4}$ on the vertices of our tetrahedron: for example,

$$
\begin{aligned}
\operatorname{av}\left(r_{21}\right) & =\left(r_{21}+r_{31}+r_{32}+r_{41}+r_{42}+r_{43}\right) / 6 \\
\operatorname{av}\left(r_{21} r_{43}\right) & =\left(r_{21} r_{43}+r_{31} r_{42}+r_{41} r_{32}\right) / 3 .
\end{aligned}
$$

The final two terms are non-negative since the triangle inequality gives

$$
\left(r_{42}+r_{43}\right)^{2} \geq r_{32}^{2} \quad \text { and } \quad d_{3}\left(r_{21}, r_{31}, r_{32}\right) \geq 0
$$

and the square of the volume is non-negative. To estimate the other terms we may use the easily verified inequality

$$
a b c \geq d_{3}(a, b, c), \quad \forall a, b, c \geq 0 .
$$

In conclusion,

$$
\Re(\operatorname{det} M) \geq 60 r_{21} r_{31} r_{32} r_{41} r_{42} r_{43}>0,
$$

as required. This is nearly enough for a stronger conjecture of Atiyah and Sutcliffe [3, Conjecture 2] that $|\operatorname{det} M| \geq 64 r_{21} r_{31} r_{32} r_{41} r_{42} r_{43}$.

\footnotetext{
\#The program is at: ftp://ftp.maths.adelaide.edu.au/meastwood/maple/points
} 
A third conjecture of Atiyah and Sutcliffe [3, Conjecture 3] can be expressed in the four point case in terms of polynomials in the edge lengths as:

$$
|\operatorname{det} M|^{2} \geq \prod_{1}^{4}\left(d_{3}\left(r_{i j}, r_{i k}, r_{j k}\right)+8 r_{i j} r_{i k} r_{j k}\right)
$$

where the product runs over the four faces of the tetrahedron and the left hand side is known explicitly. We have been unable to prove this conjecture even in the case that the four points lie in a plane (in which case $|\operatorname{det} M|$ can be replaced by the simpler expression $\Re(\operatorname{det} M)$ given above).

\section{The planar case}

Atiyah's basic conjecture is unresolved in general, even when the $n$ points lie in a plane (in which case recall that $\operatorname{det} M$ is real). Reasoning analogous to the case of four points gives the following.

Theorem 2 The Atiyah determinant of $n$ points in a plane can be expressed as a rational function in the distances between the points.

Proof Again, we can express $z_{i j} \bar{z}_{k l}$ as a rational function in the $r_{i j}$ and $A_{i j k}$. It is no longer true that quadratic expressions in the $A_{i j k}$ are polynomials in the $r_{i j}$. Instead they are rational functions in the $r_{i j}$. This uses the same trick of introducing new points in common between two triangles in order to apply (4):

$$
A_{i j k} A_{l m n}=\frac{\left(A_{i j k} A_{i j n}\right)\left(A_{i m n} A_{l m n}\right)}{A_{i j n} A_{i m n}} .
$$

In the general four point case, the distances $r_{i j}$ acted as variables. The denominator was too small to be appropriately symmetrical and therefore had to divide the numerator, leaving a polynomial rather than a rational function. In the planar case (and also in the general case with more than four points), the distances between points satisfy a set of polynomial constraints. Symmetry arguments are no longer valid and expressions for the determinant are no longer unique. We suspect, however, that there is a polynomial expression.

Acknowledgements Support from the Australian Research Council and the Mathematical Sciences Research Institute is gratefully acknowledged. Research at MSRI is supported in part by NSF grant DMS-9701755. 


\section{References}

[1] MF Atiyah, The geometry of classical particles, Surveys in Differential Geometry vol. 7, International Press (2001) to appear

[2] M F Atiyah, Configurations of points, Phil. Trans. Roy. Soc. Lond. A359 (2001) to appear

[3] M F Atiyah, P M Sutcliffe, The geometry of point particles, hep-th/0105179 